8. M. Nakaoka and H. Toda, On Jacobi identity for Whitehead products, J. Inst. Polytech. Osaka City Univ. Ser. A. 5 (1954), 1-13.

9. J.-P. Serre, Groupes d'homotopie et classes de groupes abeliens, Ann. of Math. (2) 58 (1953), 258-294.

10. N. Shimada, Triviality of the mod $p$ Hopf invariants, Proc. Japan Acad. 36 (1960), 68-69.

11. N. E. Steenrod, Cohomology invariants of mappings, Ann. of Math. (2) 50 (1949), 954-988.

12. H. Toda, On double suspension $E^{2}$, J. Inst. Polytech. Osaka City Univ. Ser A. 7 (1956), 103-146.

13. - p-primary components of homotopy groups of spheres. II, mod $p$ Hopf invariant, Memoirs Univ. Kyoto 31 (1958), 143-160.

14. - p-primary components of homotopy groups of spheres. IV, Toric constructions, Memoirs Univ. Kyoto 32 (1959), 297-332.

15. Composition methods in homotopy groups of spheres, Annals of Math. Studies 49, Princeton (to appear).

16. G. W. Whitehead, A generalization of the Hopf invariant, Ann. of Math. (2) 51 (1950), 192-237.

NORTHWESTERN UNIVERSITY AND

UNIVERSITY OF KYOTO

\title{
A NOTE ON SUBGROUPS OF THE MODULAR GROUP1
}

\section{MARVIN ISADORE KNOPP}

1. We will follow the notation of [4]. Let $\Gamma$ denote the $2 \times 2$ modular group, that is, the set of all $2 \times 2$ matrices with rational integral entries and determinant 1 . For each positive integer $m$ define $\Gamma(m)$, the principal congruence subgroup of level $m$, by

$$
\Gamma(m)=\left\{X=\left(\begin{array}{ll}
a & b \\
c & d
\end{array}\right) \in \Gamma \mid a \equiv d \equiv 1, b \equiv c \equiv 0(\bmod m)\right\} .
$$

Let

$$
T_{m}=\left(\begin{array}{cc}
1 & m \\
0 & 1
\end{array}\right)
$$

and let $\Delta(m)$ be the normal subgroup of $\Gamma$ generated by $T_{m}$. That is, $\Delta(m)$ is the smallest normal subgroup of $\Gamma$ containing $T_{m}$. Clearly, $\Delta(m) \subset \Gamma(m)$.

In [4] Reiner considers the following questions raised in [1]:

Received by the editors October 6, 1961.

${ }^{1}$ Research supported in part by National Science Foundation Grant No. G-14362 at the University of Wisconsin, Madison. 
(a) Does $\Delta(m)=\Gamma(m)$ for all $m$ ?

(b) For each $m$, does there exist a positive integer $k$ such that $\Delta(m) \supset \Gamma(m k)$ ?

He answers both questions in the negative by proving that if $m>1$ and $m$ is not a prime power, then $\Delta(m)$ does not contain any principal congruence subgroup. The situation when $m$ is a prime power is left open.

The purposes of this note are to point out that the following result is to be found (at least implicitly) in [2] and to give a new proof.

TheOREM. If $m \geqq 6$, then $\Delta(m)$ is of infinite index in $\Gamma(m)$. Since a principal congruence is always of finite index in $\Gamma$, it is a consequence that for $m \geqq 6, \Delta(m)$ contains no principal congruence subgroup. [4].

I would like to thank Dr. J. R. Smart for calling my attention to

We will make use of the following simple

LeмmA. $\Delta(m)$ is generated by the set

$$
H=\left\{X^{-1} T_{m} X \mid X \in \Gamma\right\} .
$$

The proof is obtained by noting that the group $G$ generated by $H$ is normal in $\Gamma$ and that $G \subset \Delta(m)$.

Using this lemma we obtain from $[2$, pp. $267,354-356]$ that $\Delta(m)$ $=\Gamma(m)$, for $1 \leqq m \leqq 5$, and from $[2$, pp. 356-360] that $\Delta(m)$ is of infinite index in $\Gamma(m)$, for $m \geqq 6$.

2. We now give an independent proof of this latter fact based upon the results of [3]. In [3] it was shown that given $m \geqq 2$ there exists a function, say $\lambda_{1}(m ; \tau)$, defined and analytic in $g(\tau)>0$ such that

(i) $\lambda_{1}(m ; X \tau)=\lambda_{1}(m ; \tau)+c(X)$, for each $X \in \Gamma(m)$ and for $g(\tau)>0$, where $c(X)$ is independent of $\tau$;

(ii) $c(X)=0$, when

$$
X=\left(\begin{array}{ll}
a & b \\
c & d
\end{array}\right)
$$

is parabolic, that is, when $|a+d|=2$;

(iii) $\lambda_{1}(m ; \tau)$ has a pole of order 1 in the local uniformizing variable at one of the parabolic cusps of $\mathcal{F}_{m}$, the fundamental region of $\Gamma(m)$, and is regular at all of the other parabolic cusps of $\mathcal{F}_{m}$.

Of course, (i) and (iii) show that $\lambda_{1}(m ; \tau)$ is an abelian integral connected with $\Gamma(m)$. 
Now $T_{m}$ is parabolic and a simple computation shows that each element of $H$ is parabolic. But $c\left(X_{1} X_{2}\right)=c\left(X_{1}\right)+c\left(X_{2}\right)$ and by (ii) $c(X)$ $=0$, for $X \in \Delta(m)$. Thus for $X \in \Gamma(m), c(X)$ depends only upon the coset of $X$ modulo $\Delta(m)$. Thus if $\Delta(m)$ has finite index in $\Gamma(m)$, there are only finitely many distinct values $c(X)$, with $X \in \Gamma(m)$. But this implies that $c(X)=0$ for all $X \in \Gamma(m)$. For if there exists $X_{0} \in \Gamma(m)$, with $c\left(X_{0}\right) \neq 0$, then $c\left(X_{0}^{t}\right)=t c\left(X_{0}\right) ; t=1,2,3, \cdots$ provides us with infinitely many distinct values $c(X)$.

Thus if $\Delta(m)$ has finite index in $\Gamma(m), \lambda_{1}(m ; \tau)$ is an invariant with respect to $\Gamma(m)$ with precisely one pole of order 1 in $\mathcal{F}_{m}$. Since $\mathcal{F}_{m}$ is compact, the Riemann-Roch Theorem implies that the genus of $\mathcal{F}_{m}$ is zero. By $\left[2\right.$, p. 398] $\mathcal{F}_{m}$ has genus zero exactly when $1 \leqq m \leqq 5$. Thus for $m \geqq 6, \Delta(m)$ has infinite index in $\Gamma(m)$.

The proof shows that when $m \geqq 6, \lambda_{1}(m ; \tau)$ cannot be invariant with respect to any subgroup of finite index in $\Gamma(m)$.

\section{REFERENCES}

1. J. L. Brenner, The linear homogeneous group. III, Ann. of Math. (2) 71 (1960), 210-223.

2. F. Klein and R. Fricke, Vorlesungen über die Theorie der elliptischen Modulfunktionen, Vol. 1, Teubner, Leipzig, 1890-1892.

3. M. I. Knopp, Construction of a class of modular functions and forms. II, Pacific J. Math. 11 (1961), 661-678.

4. I. Reiner, Subgroups of the unimodular group, Proc. Amer. Math. Soc. 12 (1961), 173-174.

ThE University OF Wisconsin 